\title{
All-Carbon-Substituted Vinylsilane Stable to TBAF: Synthesis of Allyldimethylvinylsilane and Its Pd-Catalyzed Cross-Coupling under Mild Conditions
}

\author{
Lianhai Li* and Neenah Navasero \\ Department of Medicinal Chemistry, Merck Frosst Centre for Therapeutic Research \\ PO Box 1005, Pointe-Claire-Dorval, Quebec, Canada H9R 4P8. \\ Lianhai_li@merck.com
}

\section{General Procedure:}

\section{$\underline{\text { General Experimental }}$}

Unless otherwise stated, reactions were performed under a nitrogen atmosphere using commercially obtained anhydrous solvents as received. Alkyllithium reagents were titrated with 1,10-phenanthroline as an indicator in ether. ${ }^{1}$ All other commercially obtained reagents were used as received. All reactions were magnetically stirred and monitored by thin-layer chromatography (TLC) using E. Merck silica gel 60 F254 precoated plates $(0.25-\mathrm{mm})$. Column or flash chromatography was performed with the indicated solvents using silica gel (particle size 0.032-0.063 nm) purchased from Bodman. ${ }^{1} \mathrm{H}$ and ${ }^{13} \mathrm{C}$ NMR were recorded on Bruker Advance DPX-500 using acetone- $d_{6}$ as the solvent. Chemical shifts $(\delta)$ are given in parts per million (ppm) relative to internal solvent (acetone- $d 6:{ }^{1} \mathrm{H} @ 2.05$ ppm, ${ }^{13} \mathrm{C} @ 29.84$ ppm), and coupling constants $(J)$ are given in hertz $(\mathrm{Hz})$.

\section{Experimentals}

\section{Dimethyl[bis(2-methylprop-2-en-1-yl)]silane (1b):}

\footnotetext{
${ }^{1}$ Stanetty, P.; Mihovilovic, M.D. J. Org. Chem. 1997, 62, 1514-1515.
} 


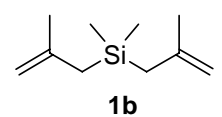

To a vigorously stirred suspension of $\mathrm{Mg}(1.5 \mathrm{~mol})$ in THF $(200 \mathrm{~mL})$ was added $1.5 \mathrm{~mL}$ of $\mathrm{BrCH}_{2} \mathrm{CH}_{2} \mathrm{Br}$ and heated until bubbling was observer. After the bubbling finished, a solution of 1-chloro-2-methyl-1-propene (1.3 mol), dichlorodimethylsilane (0.5 mol), and THF $(800 \mathrm{~mL})$ was added dropwise at the speed that kept the reaction at a gentle reflux. After the addition, the reaction mixture was allowed to reflux for $1 \mathrm{hr}$. The resulting mixture was diluted with $1 \mathrm{~L}$ of hexane at $\mathrm{rt}$ and $30 \mathrm{~mL}$ of saturated $\mathrm{NH}_{4} \mathrm{Cl}$ aqueous solution was added dropwise. The mixture was filtered then washed with water. Dried on $\mathrm{Na}_{2} \mathrm{SO}_{4}$, evaporated and distilled (bp. $\left.178-9{ }^{\circ} \mathrm{C}\right)^{2}$ to afford $80 \mathrm{~g}$ of clear liquid as the desired product (95\% yield). ${ }^{1} \mathrm{H}$ NMR $(500 \mathrm{MHz}$, Acetone): $\delta 4.58$ (m, $2 \mathrm{H}) ; 4.48$ (s, $2 \mathrm{H}) ; 1.70$ (m, $6 \mathrm{H}) ; 1.59$ (d, J = 0.8 Hz, $4 \mathrm{H}) ; 0.05$ (d, J = 3.2 $\mathrm{Hz}, 6 \mathrm{H}) .{ }^{13} \mathrm{C}$ NMR (500 MHz, Acetone): $\delta 144.11,108.98,27.55,25.42,-2.68$.

\section{(3E)-4-[allyl(dimethyl)silyl]-1-phenylbut-3-en-1-ol (3a):}

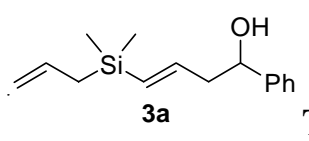

To a stirred solution of diallyldimethylsilane $(1.00 \mathrm{~mL}, 5.5 \mathrm{mmol}, 1.1 \mathrm{eq})$ and $\mathrm{KO}^{\mathrm{t}} \mathrm{Bu}(1.0 \mathrm{M}$ THF, $5.0 \mathrm{~mL}, 5 \mathrm{mmol}, 1 \mathrm{eq})$ in $30 \mathrm{~mL}$ THF at $-78^{\circ} \mathrm{C}, n$-BuLi $(2.5 \mathrm{M}$ in hexanes, $2.0 \mathrm{~mL}, 5.0 \mathrm{mmol}, 1 \mathrm{eq}$ ) was added dropwise. The resulting mixture was stirred at $-78{ }^{\circ} \mathrm{C}$ for $1 \mathrm{hr}$. Then benzaldehyde $(0.51 \mathrm{~mL}, 5.0 \mathrm{mmol}, 1 \mathrm{eq})$ was added dropwise and the resulting solution was stirred at $-78^{\circ} \mathrm{C}$ for $0.5 \mathrm{~h}$. The reaction was quenched with the addition of sat. $\mathrm{NaHCO}_{3}$ aqueous solution and extracted with a $30 \%$ solution of EtOAc in Hexanes, dried with $\mathrm{Na}_{2} \mathrm{SO}_{4}$, filtered and evaporated. The product was purified by flash chromatography on silica gel (10\% EtOAc/hexane) to afford the desired product as colorless oil ( $0.83 \mathrm{~g}, 67 \%$ yield). ${ }^{1} \mathrm{H}$ NMR (500 MHz, Acetone): $\delta 7.35$ (d, $J=7.1 \mathrm{~Hz}, 2 \mathrm{H}) ; 7.30$ (t, $J=7.4 \mathrm{~Hz} 2 \mathrm{H}) ; 7.21(\mathrm{t}, J=7.2 \mathrm{~Hz}, 1 \mathrm{H})$; $6.12(\mathrm{dt}, J=18.7,6.6 \mathrm{~Hz}, 1 \mathrm{H}) ; 5.77-5.69(\mathrm{~m}, 1 \mathrm{H}) ; 5.63(\mathrm{~d}, J=18.7 \mathrm{~Hz} 1 \mathrm{H}) ; 4.81-4.71(\mathrm{~m}, 3$ $\mathrm{H}) ; 4.22(\mathrm{~d}, J=4.1 \mathrm{~Hz}, 1 \mathrm{H}) ; 2.57-2.45(\mathrm{~m}, 4 \mathrm{H}) ; 1.52(\mathrm{~d}, J=8.1 \mathrm{~Hz}, 2 \mathrm{H}) ; 0.01(\mathrm{~s}, 6 \mathrm{H}) .{ }^{13} \mathrm{C}$ NMR (500 MHz, Acetone): $\delta 146.39,145.65,135.82,131.17,128.73,127.59,126.74,113.15$, $73.86,48.09,24.16,-3.37$.

\section{(5E)-6-[allyl(dimethyl)silyl]-1-phenylhex-5-en-3-ol (3d):}

\footnotetext{
${ }^{2}$ For literature boiling point see: Nasiak, L. D.; Post, H. W. J. Org. Chem. 1959, 24, 489-96 and references therein.
} 


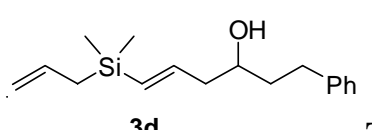

To a stirred solution of diallyldimethylsilane $(0.2 \mathrm{~mL}, 1.1 \mathrm{mmol}, 1.1 \mathrm{eq})$ and $\mathrm{KO}{ }^{t} \mathrm{Bu}(1.0 \mathrm{M}$ THF, $1.0 \mathrm{~mL}, 1 \mathrm{mmol}, 1 \mathrm{eq})$ in $6 \mathrm{~mL}$ THF at $-78^{\circ} \mathrm{C}, n-\mathrm{BuLi}(2.5 \mathrm{M}$ in hexanes, $0.4 \mathrm{~mL}, 1.0 \mathrm{mmol}, 1 \mathrm{eq}$ ) was added dropwise. The resulting mixture was stirred at -78 ${ }^{\circ} \mathrm{C}$ for $1 \mathrm{hr}$. Then $\mathrm{CuCN}$ (109 mg, $1.2 \mathrm{mmol}, 1.2 \mathrm{eq}$ ) was added at $-78^{\circ} \mathrm{C}$. After stirred for $0.5 \mathrm{~h}$, to the resulting mixture was added hydrocinnamaldehyde $(145 \mu \mathrm{L}, 1.0 \mathrm{mmol}, 1 \mathrm{eq})$ dropwise and the resulting mixture was stirred at $-78^{\circ} \mathrm{C}$ for $0.5 \mathrm{~h}$ and at $\mathrm{rt}$ for $12 \mathrm{hr}$. The reaction was quenched with the addition of water and extracted with EtOAc, dried with $\mathrm{Na}_{2} \mathrm{SO}_{4}$, filtered and evaporated. The crude was purified by flash chromatography on silica gel (10\% EtOAc/hexane) to afford the desired product as colorless oil (162 mg, 59\% yield). ${ }^{1} \mathrm{H}$ NMR (500 MHz, Acetone): $\delta 7.25$ (t, $J=$ $7.4 \mathrm{~Hz}, 2 \mathrm{H}) ; 7.20$ (d, $J=6.9 \mathrm{~Hz}, 2 \mathrm{H}) ; 7.14(\mathrm{t}, J=7.2 \mathrm{~Hz}, 1 \mathrm{H}) ; 6.18(\mathrm{dt}, J=18.6,6.7 \mathrm{~Hz}, 1$ $\mathrm{H}) ; 5.69(\mathrm{dt}, J=18.7,1.4 \mathrm{~Hz}, 1 \mathrm{H}) ; 4.84-4.75(\mathrm{~m}, 2 \mathrm{H}) ; 3.68-3.60(\mathrm{~m}, 2 \mathrm{H}) ; 2.81-2.75(\mathrm{~m}, 1$ $\mathrm{H}) ; 2.69-2.61$ (m, $1 \mathrm{H}) ; 2.33-2.29$ (m, $2 \mathrm{H}) ; 1.79-1.63(\mathrm{~m}, 2 \mathrm{H}) ; 1.55(\mathrm{~d}, J=8.1 \mathrm{~Hz}, 2 \mathrm{H}) ; 0.03$ (s, $6 \mathrm{H}) .{ }^{13} \mathrm{C}$ NMR (500 MHz, Acetone): $\delta 146.07,143.55,135.85,130.89,129.19,129.06$, $126.37,113.18,70.32,46.16,39.70,32.57,24.23,-3.30$.

\section{(4E)-5-[allyl(dimethyl)silyl]-2-methylpent-4-en-2-ol (3b):}

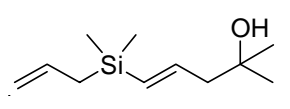

3b $\quad$ To a stirred solution of diallyldimethylsilane $(2.00 \mathrm{~mL}, 11 \mathrm{mmol}, 1.1 \mathrm{eq})$ and $\mathrm{KO}^{\mathrm{t}} \mathrm{Bu}(1.0 \mathrm{M} \mathrm{THF}, 10.0 \mathrm{~mL}, 10 \mathrm{mmol}, 1 \mathrm{eq})$ in $60 \mathrm{~mL}$ THF at $-78^{\circ} \mathrm{C}, n-\mathrm{BuLi}(2.5 \mathrm{M}$ in hexanes, $4 \mathrm{~mL}, 10 \mathrm{mmol}, 1 \mathrm{eq}$ ) was added dropwise. The resulting mixture was stirred at $-78{ }^{\circ} \mathrm{C}$ for $1 \mathrm{hr}$, and was added $\mathrm{ZnCl}_{2}(1.0 \mathrm{M}$ in ether, $10 \mathrm{~mL}, 10 \mathrm{mmol}, 1 \mathrm{eq})$ and stired for an additional 10 minutes at $-78^{\circ} \mathrm{C}$. Then acetone $(0.81 \mathrm{~mL}, 10 \mathrm{mmol}, 1.1 \mathrm{eq})$ was added dropwise. The resulting mixture was stirred at $-78{ }^{\circ} \mathrm{C}$ for $0.5 \mathrm{~h}$ and then at $0{ }^{\circ} \mathrm{C}$ for $1 \mathrm{~h}$. The reaction was quenched with the addition of a sat. $\mathrm{NaHCO}_{3}$ aqueous solution was extracted with a $30 \%$ solution of EtOAc in Hexanes, dried with $\mathrm{Na}_{2} \mathrm{SO}_{4}$, filtered and evaporated. The residue was purified by flash chromatography on silica gel (10\% EtOAc/hexane) to afford the desired product as colorless oil (1.07 g, 54\% yield). ${ }^{1} \mathrm{H}$ NMR (500 MHz, Acetone): $\delta 6.21(\mathrm{dt}, J=18.6,7.0 \mathrm{~Hz}, 1 \mathrm{H}) ; 5.83$ $5.73(\mathrm{~m}, 1 \mathrm{H}) ; 5.65(\mathrm{~d}, J=18.6 \mathrm{~Hz}, 1 \mathrm{H}) ; 4.85-4.75(\mathrm{~m}, 2 \mathrm{H}) ; 3.30(\mathrm{~s}, \mathrm{H}) ; 2.27$ (d, J=6.9 Hz, 2 $\mathrm{H}) ; 1.57(\mathrm{~d}, J=8.1 \mathrm{~Hz}, 2 \mathrm{H}) ; 1.13(\mathrm{~s}, 6 \mathrm{H}) ; 0.05(\mathrm{~s}, 6 \mathrm{H}) .{ }^{13} \mathrm{C}$ NMR (500 MHz, Acetone): $\delta 145.96,135.87,131.49,113.14,69.98,52.42,29.52,24.25,-3.30$. 


\section{1-\{(2E)-3-[allyl(dimethyl)silyl]prop-2-en-1-yl $\}$ cyclohexanol (3c):}

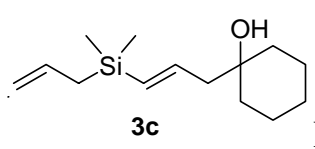

Followed the procedure above and used cyclohexanone to replace acetone to perform the reaction, the desired compound was obtained (1.17 g, 52\% yield). 1H NMR (500 MHz, Acetone): $\delta 6.24(\mathrm{dt}, J=18.6,6.9 \mathrm{~Hz}, 1 \mathrm{H}) ; 5.82-5.74(\mathrm{~m}, 1 \mathrm{H}) ; 5.65(\mathrm{~d}, J=18.6 \mathrm{~Hz}, 1 \mathrm{H})$; 4.83-4.76 (m, $2 \mathrm{H}) ; 3.05$ (s, $1 \mathrm{H} \mathrm{OH}) ; 2.25(\mathrm{dd}, J=1.2,6.9 \mathrm{~Hz}, 2 \mathrm{H}) ; 1.67-1.59$ (m, $2 \mathrm{H}) ; 1.56$ $(\mathrm{d}, J=8.0 \mathrm{~Hz}, 2 \mathrm{H}) ; 1.53-1.45$ (m, $3 \mathrm{H}) ; 1.42-1.34(\mathrm{~m}, 4 \mathrm{H}) ; 1.27-1.17$ (m, $1 \mathrm{H}) ; 0.04(\mathrm{~s}, 6 \mathrm{H})$. 13C NMR (500 MHz, Acetone): 8145.44, 135.90, 131.51, 113.11, 70.76, 51.41, 38.10, 26.60, $24.30,22.73,-3.28$.

(3E)-4-[dimethyl(2-methylprop-2-en-1-yl)silyl]-3-methyl-1-phenylbut-3-en-1-ol (3e):<smiles>C=C(C)C[Si](C)(C)C=C(C)CC(O)c1ccccc1</smiles>

To a stirred solution of dimethyl[bis(2-methylprop-2-en-1-yl)]silane (1b) $(2.35 \mathrm{~mL}, 11 \mathrm{mmol}, 1.1 \mathrm{eq})$ and $\mathrm{KO}^{\mathrm{t}} \mathrm{Bu}(1.0 \mathrm{M} \mathrm{THF}, 10.0 \mathrm{~mL}, 10 \mathrm{mmol}, 1 \mathrm{eq})$ in $60 \mathrm{~mL}$ ether at $-78^{\circ} \mathrm{C}, n$-BuLi (2.5 $\mathrm{M}$ in hexanes, $\left.4 \mathrm{~mL}, 10 \mathrm{mmol}, 1 \mathrm{eq}\right)$ was added dropwise. The resulting mixture was stirred at $-78{ }^{\circ} \mathrm{C}$ for $1 \mathrm{hr}$, and was warmed to $0^{\circ} \mathrm{C}$ and stirred at this temperature for 1 $\mathrm{hr}$. Then the reaction mixture was cooled down to $-78^{\circ} \mathrm{C}$ and benzaldehyde $(1.11 \mathrm{~mL}, 11 \mathrm{mmol}$, $1.1 \mathrm{eq}$ ) was added dropwise and the resulting solution was stirred at $-78^{\circ} \mathrm{C}$ for $0.5 \mathrm{~h}$. The reaction was quenched with the addition of sat. $\mathrm{NaHCO}_{3}$ aqueous solution and extracted with a $30 \%$ solution of EtOAc in Hexanes, dried with $\mathrm{Na}_{2} \mathrm{SO}_{4}$, filtered and evaporated. The product was purified by flash chromatography on silica gel (10\% EtOAc/hexane) to afford the desired product as colorless oil (1.73 g, 63\% yield). ${ }^{1} \mathrm{H}$ NMR (500 MHz, Acetone): $\delta 7.35$ (d, $J=7.2 \mathrm{~Hz}, 2 \mathrm{H}$ ); $7.29(\mathrm{t}, J=7.6 \mathrm{~Hz}, 2 \mathrm{H}) ; 7.23-7.17(\mathrm{~m}, 1 \mathrm{H}) ; 5.21(\mathrm{~s}, 1 \mathrm{H}) ; 4.84-4.80(\mathrm{~m}, 1 \mathrm{H}) ; 4.55-4.52$ (m, 1 $\mathrm{H}) ; 4.45(\mathrm{~s}, 1 \mathrm{H}) ; 4.02(\mathrm{~d}, J=4.0 \mathrm{~Hz}, 1 \mathrm{H} \mathrm{OH}) ; 2.49(\mathrm{dd}, J=7.9,13.2 \mathrm{~Hz}, 1 \mathrm{H}) ; 2.42(\mathrm{dd}, J=$ 5.2, $13.2 \mathrm{~Hz}, 1 \mathrm{H}) ; 1.83$ (s, $3 \mathrm{H}) ; 1.66(\mathrm{~s}, 3 \mathrm{H}) ; 1.59$ (s, $2 \mathrm{H}) ; 0.09$ (d, J=3.7 Hz, $6 \mathrm{H})$.

${ }^{13} \mathrm{C}$ NMR (500 MHz, Acetone): $\delta 153.84,146.63,144.49,128.73,127.59,126.75,125.78,108.80$, $73.20,54.04,28.80,25.38,22.71,-1.18$.

\section{General procedure for the cross-coupling:}

To a solution of $\mathbf{3}(1.1 \mathrm{mmol}, 1.1 \mathrm{eq})$ in THF $(10 \mathrm{ml})$ stirred at $\mathrm{rt}$ under $\mathrm{N}_{2}$, EtOH $(0.32 \mathrm{ml}, 5.5$ mmol, $5.5 \mathrm{eq})$, TBAF 1 M THF (2.2 mL, $2.2 \mathrm{mmol}, 2.2 \mathrm{eq})$ and $\operatorname{Pd}_{2}(\mathrm{dba})_{3}$ or $(\operatorname{AllylPdCl})_{2}(0.05$ mmol, 0.05 eq) was added. The resulting mixture was stirred at $\mathrm{rt}$ for $0.5 \mathrm{~h}$ and to which was 
added aryl iodide ( $1 \mathrm{mmol}, 1 \mathrm{eq})$. The resulting mixture was allowed to react for $4 \mathrm{hr}$ [when $\mathrm{Pd}_{2}(\mathrm{dba})_{3}$ was used as catalyst] or $24 \mathrm{hr}$ [when $(\mathrm{AllylPdCl})_{2}$ was used as catalyst]. The reaction was worked up by the addition of water. Extracted with EtOAc, dried over $\mathrm{Na}_{2} \mathrm{SO}_{4}$, filtered and evaporated, the residue was purified by flash chromatography on silica gel to afford the desired product. $^{3}$

\section{(3E)-3-methyl-1,4-diphenylbut-3-en-1-ol (4f)}

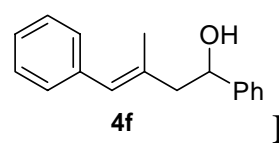

By following the general procedure described above, $\mathbf{4 f}$ was obtained as a colorless oil (177 mg , 74\% yield). ${ }^{1} \mathrm{H}$ NMR (500 MHz, Acetone): $\delta 7.42$ (d, $J=7.3 \mathrm{~Hz}$, $2 \mathrm{H})$; 7.33-7.27 (m, $4 \mathrm{H})$; 7.23-7.15 (m, $4 \mathrm{H}) ; 6.24$ (s, $1 \mathrm{H}) ; 4.92$ (ddd, J = 4.1, 5.2, 8.2 Hz, 1 $\mathrm{H}) ; 4.20(\mathrm{~d}, J=4.1 \mathrm{~Hz}, 1 \mathrm{H}) ; 2.57(\mathrm{dd}, J=8.2,13.3 \mathrm{~Hz}, 1 \mathrm{H}) ; 2.50(\mathrm{dd}, J=5.2,13.3 \mathrm{~Hz}, 1 \mathrm{H})$; $1.89(\mathrm{~d}, J=1.2 \mathrm{~Hz}, 3 \mathrm{H}) .{ }^{13} \mathrm{C}$ NMR (500 MHz, Acetone): $\delta 146.78,139.28,136.56,129.58$, $128.79,128.46,127.60,126.74,126.72,73.08,52.07,18.49$. FAB $(\mathrm{M}+\mathrm{K})^{+}$Calcd for $\mathrm{C}_{17} \mathrm{H}_{18} \mathrm{OK}$ : 277.0994; found: 277.0995 .

\section{1-\{4-[(1E)-4-hydroxy-2-methyl-4-phenylbut-1-en-1-yl]phenyl\}ethanone (4h)

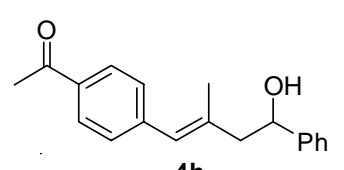

By following the general procedure described above, $4 \mathrm{~h}$ was obtained as a white solid (239 mg, $85 \%$ yield). ${ }^{1} \mathrm{H}$ NMR (500 MHz, Acetone): $\delta 7.93$ (d, $J$ $=8.3 \mathrm{~Hz}, 2 \mathrm{H}) ; 7.42(\mathrm{~d}, J=7.2 \mathrm{~Hz}, 2 \mathrm{H}) ; 7.34-7.30(\mathrm{~m}, 4 \mathrm{H}) ; 7.22(\mathrm{t}, J=7.3 \mathrm{~Hz}, 1 \mathrm{H}) ; 6.31(\mathrm{~s}$, $1 \mathrm{H}) ; 4.96-4.94(\mathrm{~m}, 1 \mathrm{H}) ; 4.27(\mathrm{~d}, J=4.2 \mathrm{~Hz}, 1 \mathrm{H} \mathrm{OH}) ; 2.63-2.50(\mathrm{~m}, 5 \mathrm{H}) ; 1.95(\mathrm{~d}, J=1.2 \mathrm{~Hz}$, $3 \mathrm{H}) .{ }^{13} \mathrm{C}$ NMR (500 MHz, Acetone): $\delta 197.32,146.68,144.01,139.43,135.69,129.63,128.90$, $128.81,127.69,127.66,126.70,73.03,52.17,26.57,18.78$. FAB $(\mathrm{M}+\mathrm{H})^{+}$Calcd for $\mathrm{C}_{19} \mathrm{H}_{21} \mathrm{O}_{2}$ : 281.1541 ; found: 281.1542 .

\section{(3E)-4-(4-methoxyphenyl)-3-methyl-1-phenylbut-3-en-1-ol (4i)}

\footnotetext{
${ }^{3}$ For the characterization of products not presented here, see: Li, L. and Navasero, N. Org. Lett.2004, 6, 3091-94.
} 


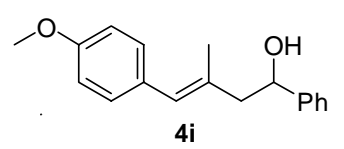

By following the general procedure described above, $\mathbf{4 i}$ was obtained as a white solid (188 mg, $70 \%$ yield). ${ }^{1} \mathrm{H}$ NMR (500 MHz, Acetone): $\delta 7.41$ (d, $J$ $=7.2 \mathrm{~Hz}, 2 \mathrm{H}) ; 7.31(\mathrm{t}, J=7.6 \mathrm{~Hz}, 2 \mathrm{H}) ; 7.21(\mathrm{t}, J=7.3 \mathrm{~Hz}, 1 \mathrm{H}) ; 7.13(\mathrm{~d}, J=8.5 \mathrm{~Hz}, 2 \mathrm{H})$; $6.86(\mathrm{~d}, J=8.7 \mathrm{~Hz}, 2 \mathrm{H}) ; 6.17(\mathrm{~s}, 1 \mathrm{H}) ; 4.90(\mathrm{ddd}, J=4.0,5.3,8.1 \mathrm{~Hz}, 1 \mathrm{H}) ; 4.15(\mathrm{~d}, J=4.0 \mathrm{~Hz}$, $1 \mathrm{H} \mathrm{OH}) ; 3.77(\mathrm{~s}, 3 \mathrm{H}) ; 2.54(\mathrm{dd}, J=8.1,13.2 \mathrm{~Hz}, 1 \mathrm{H}) ; 2.48(\mathrm{dd}, J=5.3,13.2 \mathrm{~Hz}, 1 \mathrm{H}) ; 1.88$ $(\mathrm{d}, J=1.1 \mathrm{~Hz}, 3 \mathrm{H}) .{ }^{13} \mathrm{C}$ NMR (500 MHz, Acetone): $\delta 158.87,146.83,134.74,131.72,130.72$, $128.76,128.03,127.56,126.71,114.19,73.10,55.39,52.20,18.45$. FAB $(\mathrm{M}-\mathrm{H})^{+}$Calcd for $\mathrm{C}_{18} \mathrm{H}_{19} \mathrm{O}_{2}$ : 267.1386; found: 267.1385 .

ethyl 4-[(1E)-4-hydroxy-2-methyl-4-phenylbut-1-en-1-yl]benzoate (4g)

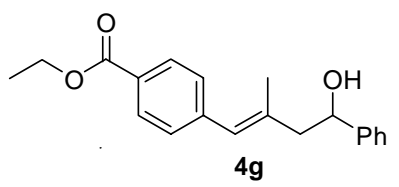

By following the general procedure described above, $\mathbf{4 g}$ was obtained as a colorless syrup (255 mg, $85 \%$ yield). ${ }^{1} \mathrm{H}$ NMR (500 MHz, Acetone): $\delta 7.95$ $(\mathrm{d}, J=8.3 \mathrm{~Hz}, 2 \mathrm{H}) ; 7.42(\mathrm{~d}, J=7.2 \mathrm{~Hz}, 2 \mathrm{H}) ; 7.32(\mathrm{t}, J=7.8 \mathrm{~Hz}, 4 \mathrm{H}) ; 7.22(\mathrm{t}, J=7.3 \mathrm{~Hz}, 1$ $\mathrm{H}) ; 6.31(\mathrm{~s}, 1 \mathrm{H}) ; 4.96-4.94(\mathrm{~m}, 1 \mathrm{H}) ; 4.32(\mathrm{q}, J=7.1 \mathrm{~Hz}, 2 \mathrm{H}) ; 4.27(\mathrm{~d}, J=4.2 \mathrm{~Hz}, 1 \mathrm{H}) ; 2.60$ $(\mathrm{dd}, J=9.0,13.3 \mathrm{~Hz}, 1 \mathrm{H}) ; 2.54(\mathrm{dd}, J=5.3,13.3 \mathrm{~Hz}, 1 \mathrm{H}) ; 1.95(\mathrm{~d}, J=1.2 \mathrm{~Hz}, 3 \mathrm{H}) ; 1.35$ (t, $J$ $=7.1 \mathrm{~Hz}, 3 \mathrm{H}) .{ }^{13} \mathrm{C}$ NMR (500 MHz, Acetone): $\delta 166.55,146.69,144.02,139.39,129.92,129.58$, 128.81, 127.66, 126.69, 73.02, 61.25, 52.14, 18.74, 14.57. FAB $(\mathrm{M}+\mathrm{H})^{+}$Calcd for $\mathrm{C}_{20} \mathrm{H}_{23} \mathrm{O}_{3}$ : 311.1648; found: 311.1647 .

\section{(3E)-4-(2-methoxyphenyl)-3-methyl-1-phenylbut-3-en-1-ol (4j)}

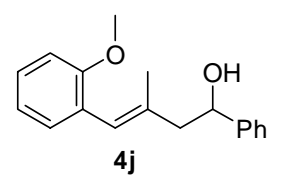

By following the general procedure described above, $4 \mathrm{~h}$ was

obtained as a colorless oil (30 mg, $11 \%$ yield). $\quad{ }^{1} \mathrm{H}$ NMR (500 MHz, Acetone): $\delta 7.42$ (d, $J$ $=7.1 \mathrm{~Hz}, 2 \mathrm{H}) ; 7.31(\mathrm{t}, J=7.6 \mathrm{~Hz}, 2 \mathrm{H}) ; 7.22-7.16(\mathrm{~m}, 2 \mathrm{H}) ; 7.13(\mathrm{~d}, J=7.5 \mathrm{~Hz}, 1 \mathrm{H}) ; 6.92(\mathrm{~d}$, $J=8.1 \mathrm{~Hz}, 1 \mathrm{H}) ; 6.88(\mathrm{t}, J=7.4 \mathrm{~Hz}, 1 \mathrm{H}) ; 6.27(\mathrm{~s}, 1 \mathrm{H}) ; 4.92-4.88(\mathrm{~m}, 1 \mathrm{H}) ; 4.09(\mathrm{~d}, J=3.9$ 
$\mathrm{Hz}, 1 \mathrm{H} \mathrm{OH}) ; 3.77(\mathrm{~s}, 3 \mathrm{H}) ; 2.58(\mathrm{dd}, J=8.0,13.2 \mathrm{~Hz}, 1 \mathrm{H}) ; 2.51(\mathrm{dd}, J=5.4,13.0 \mathrm{~Hz}, 1 \mathrm{H})$; $1.80(\mathrm{~d}, J=1.3 \mathrm{~Hz}, 3 \mathrm{H}) .{ }^{13} \mathrm{C}$ NMR (500 MHz, Acetone): $\delta 158.01,146.71,135.83,130.94$, $128.76,128.48,127.83,127.57,126.76,124.27,120.55,111.26,73.10,55.57,51.85$, 18.54. FAB (M+K) ${ }^{+}$Calcd for $\mathrm{C}_{18} \mathrm{H}_{19} \mathrm{O}_{2} \mathrm{~K}: 307.1101$; found: 307.1100 . 

and Its Pd-Catalyzed Cross-Coupling under Mild Conditions

By Lianhai Li* and Neenah Navasero
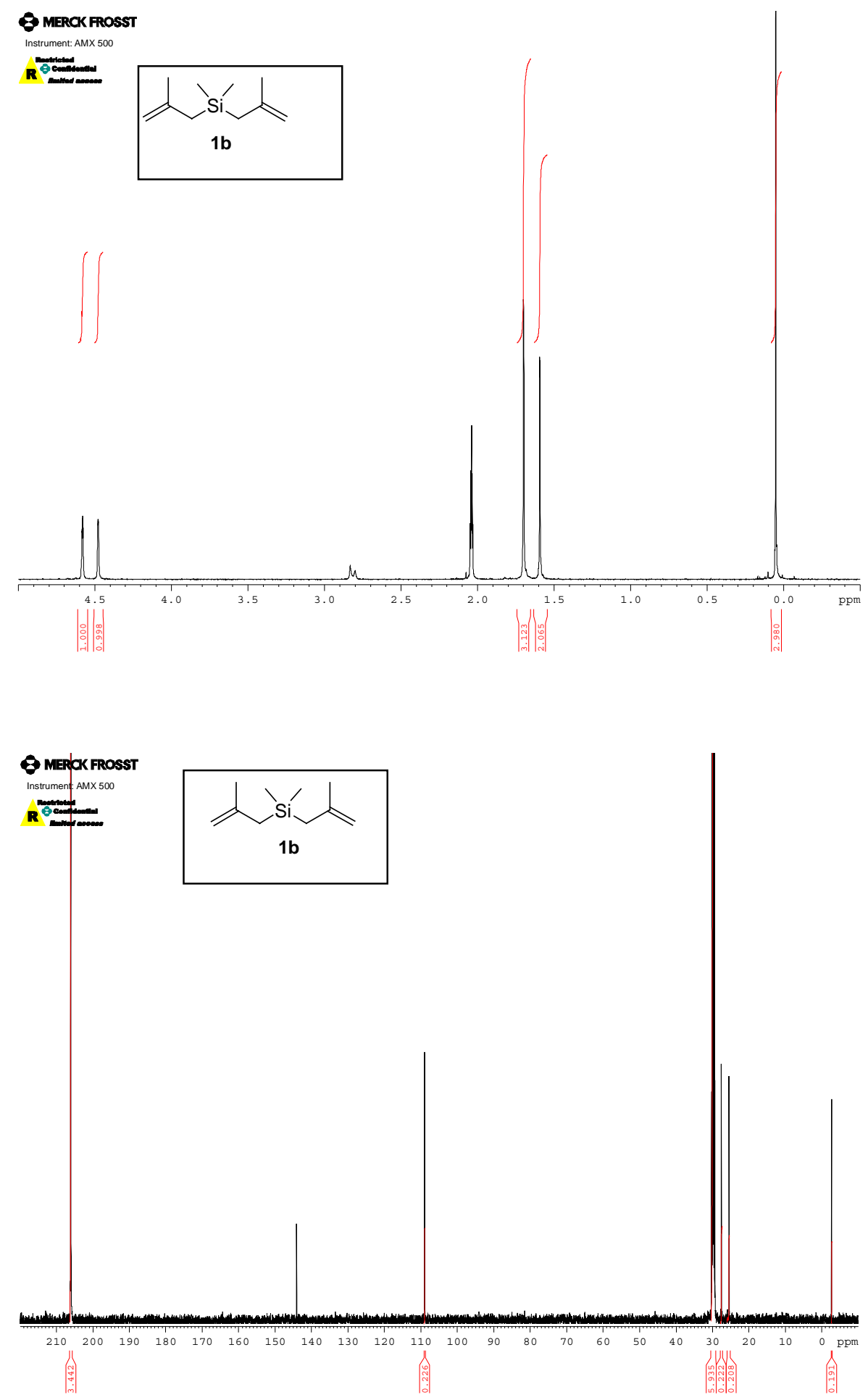

and Its Pd-Catalyzed Cross-Coupling under Mild Conditions

By Lianhai Li* and Neenah Navasero
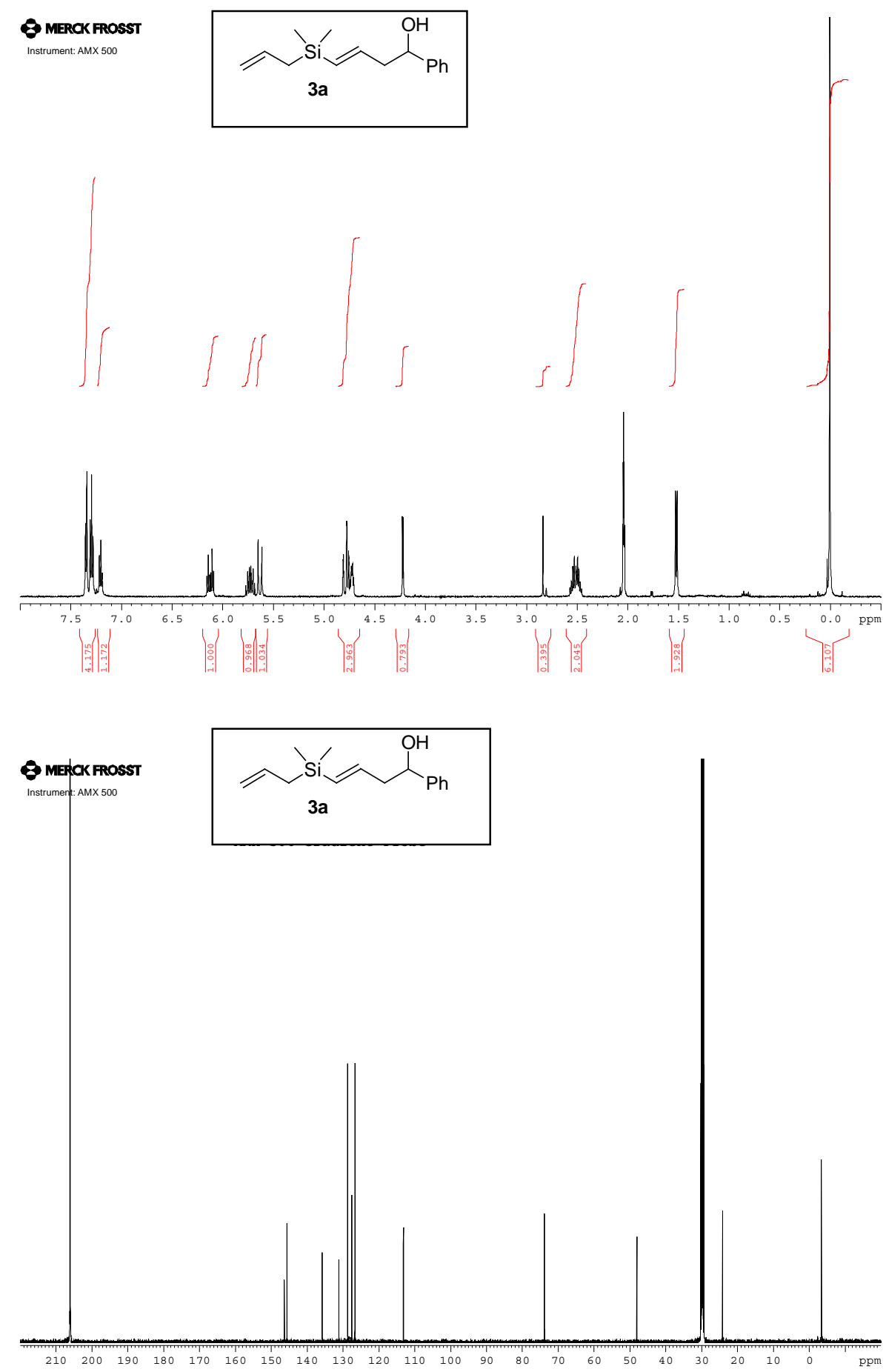

and Its Pd-Catalyzed Cross-Coupling under Mild Conditions

By Lianhai Li* and Neenah Navasero
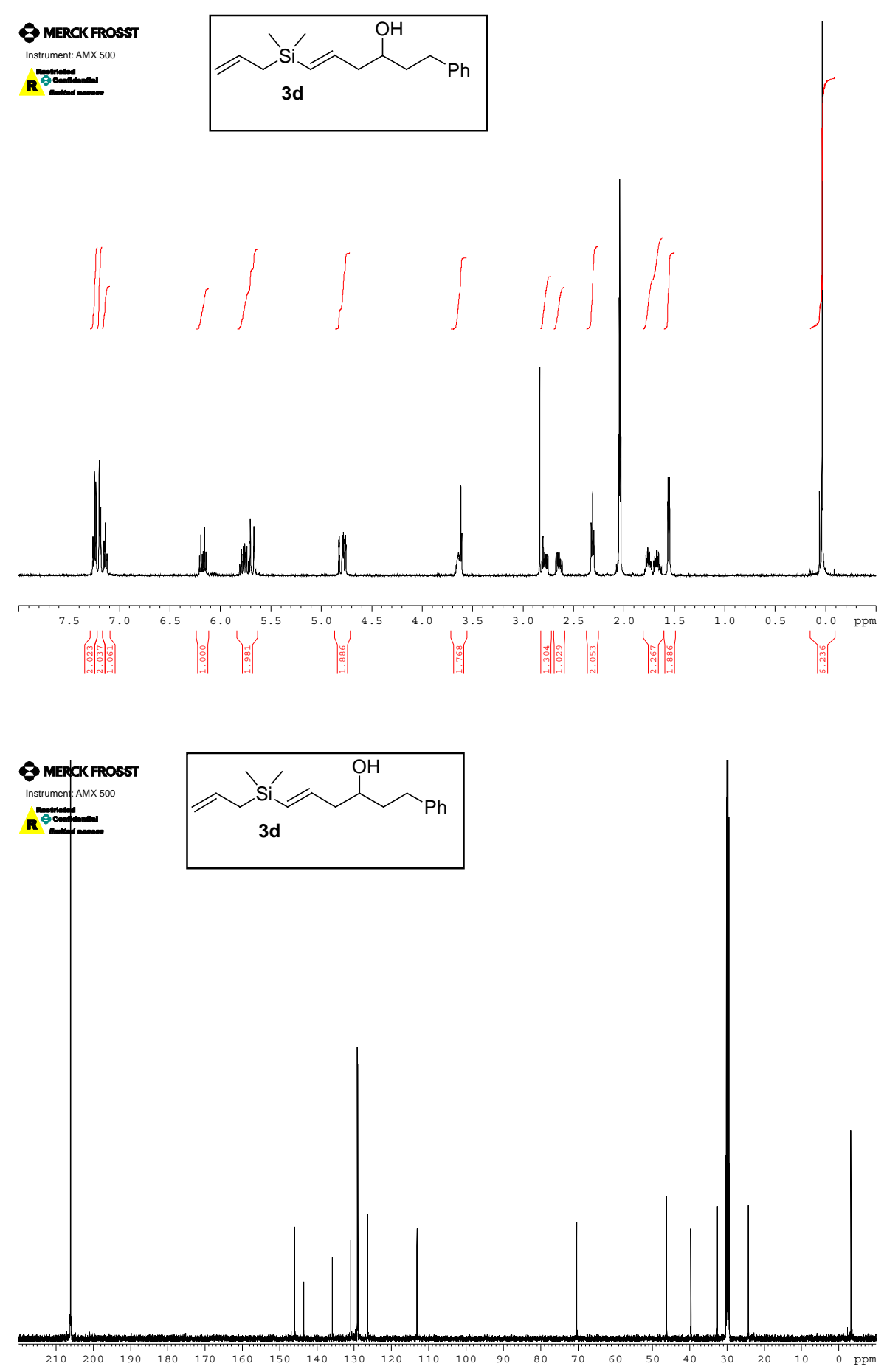

and Its Pd-Catalyzed Cross-Coupling under Mild Conditions

By Lianhai Li* and Neenah Navasero
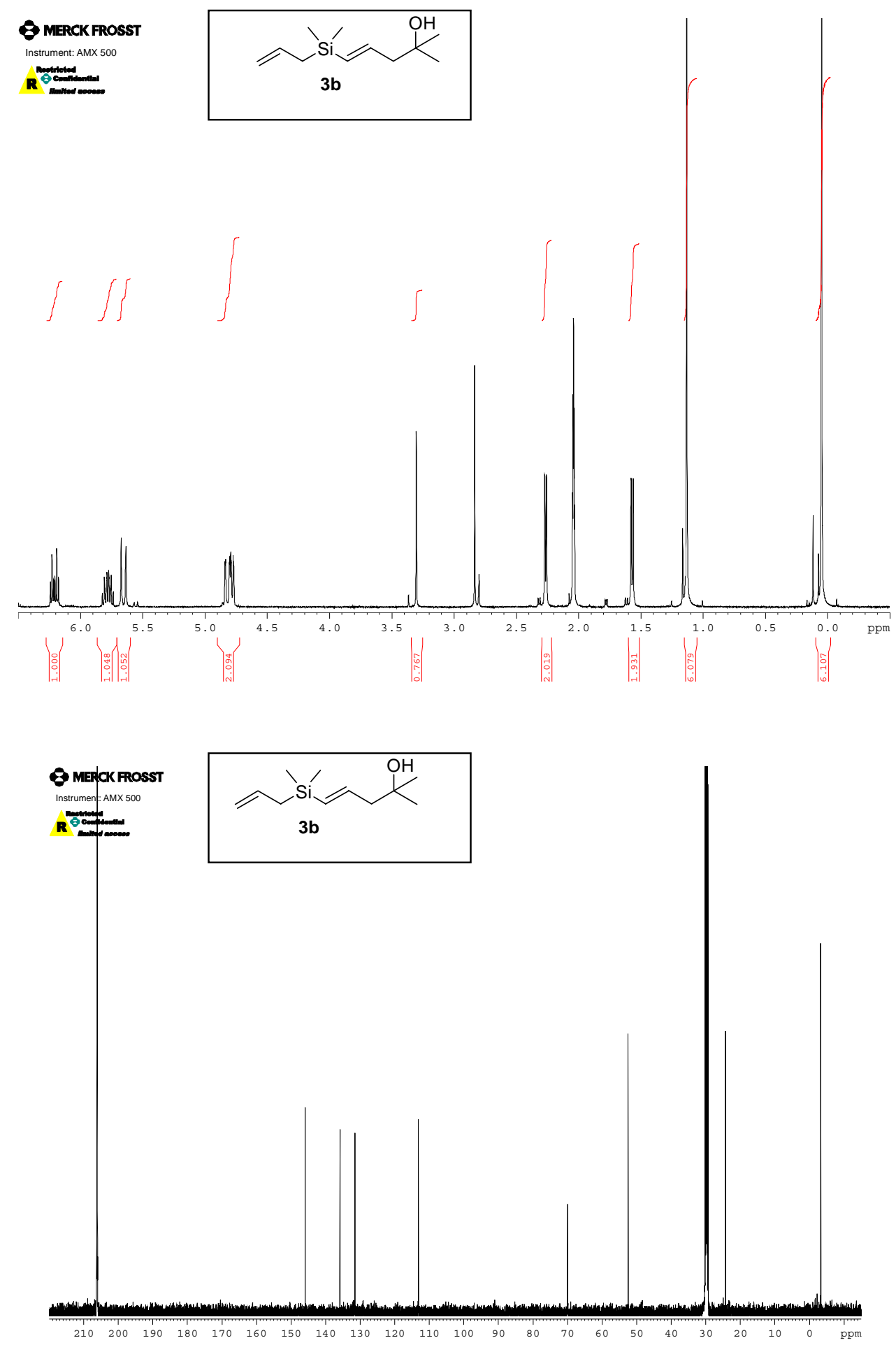

and Its Pd-Catalyzed Cross-Coupling under Mild Conditions

By Lianhai $\mathrm{Li}^{*}$ and Neenah Navasero
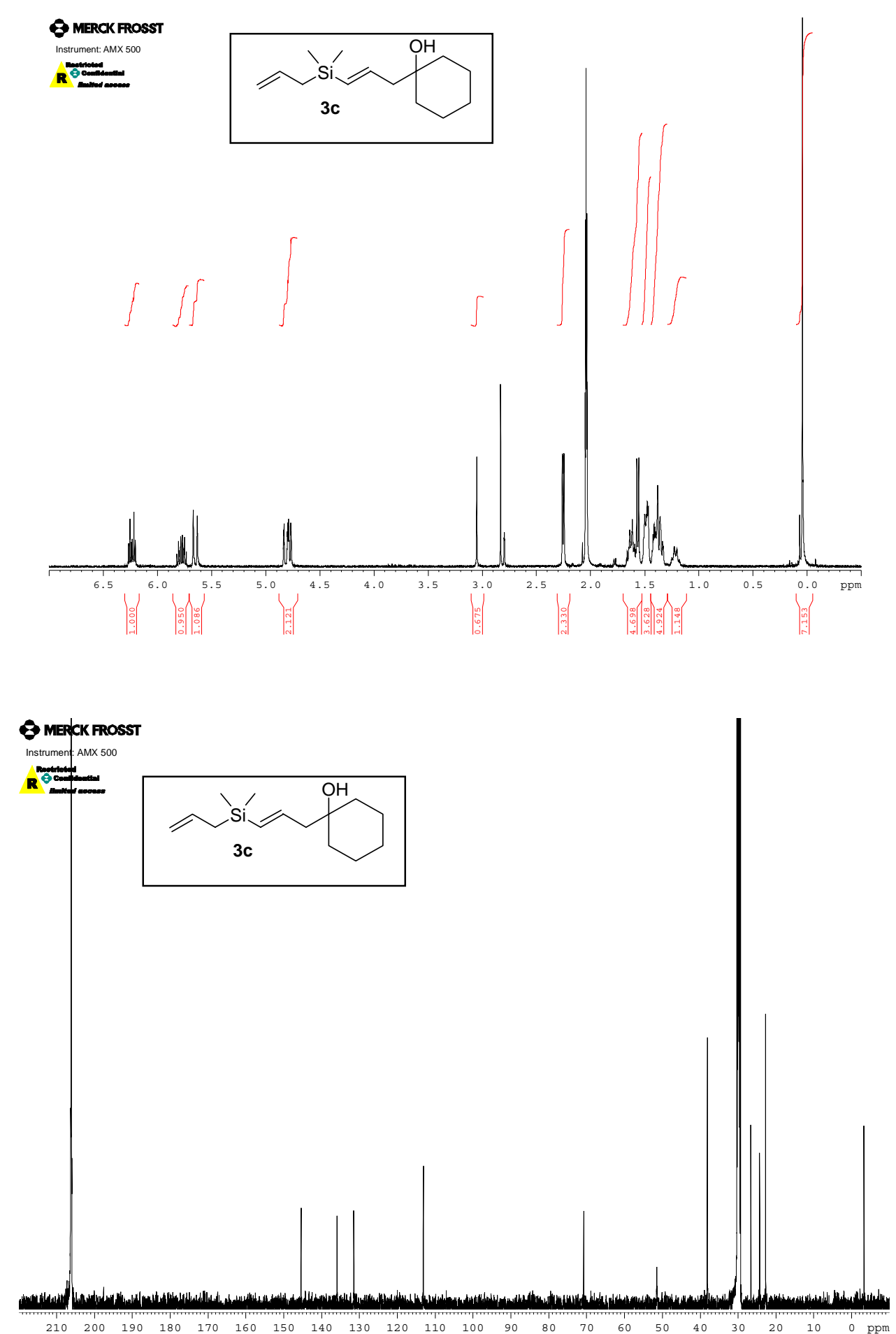

and Its Pd-Catalyzed Cross-Coupling under Mild Conditions

By Lianhai Li* and Neenah Navasero
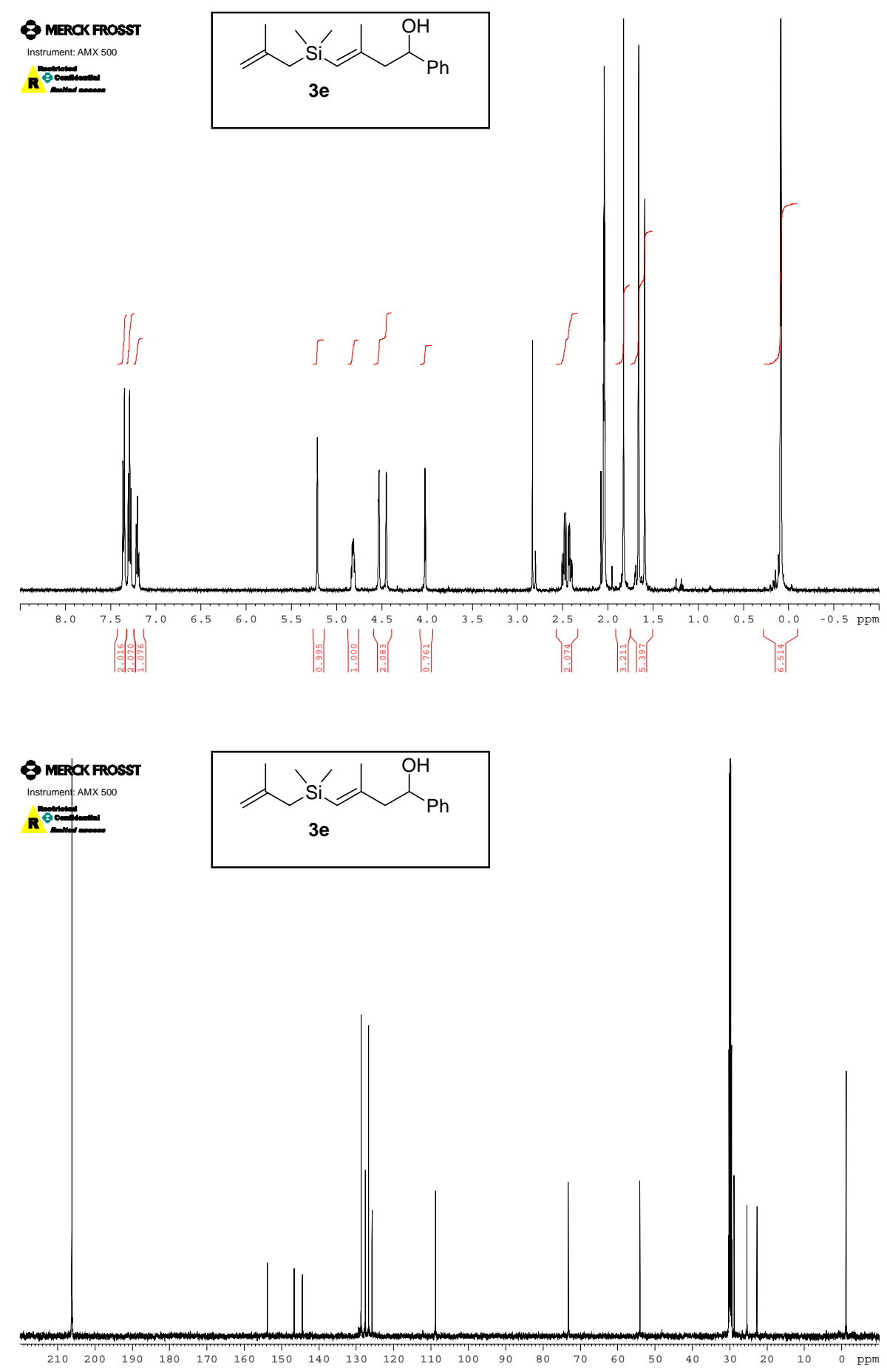

and Its Pd-Catalyzed Cross-Coupling under Mild Conditions

By Lianhai Li* and Neenah Navasero
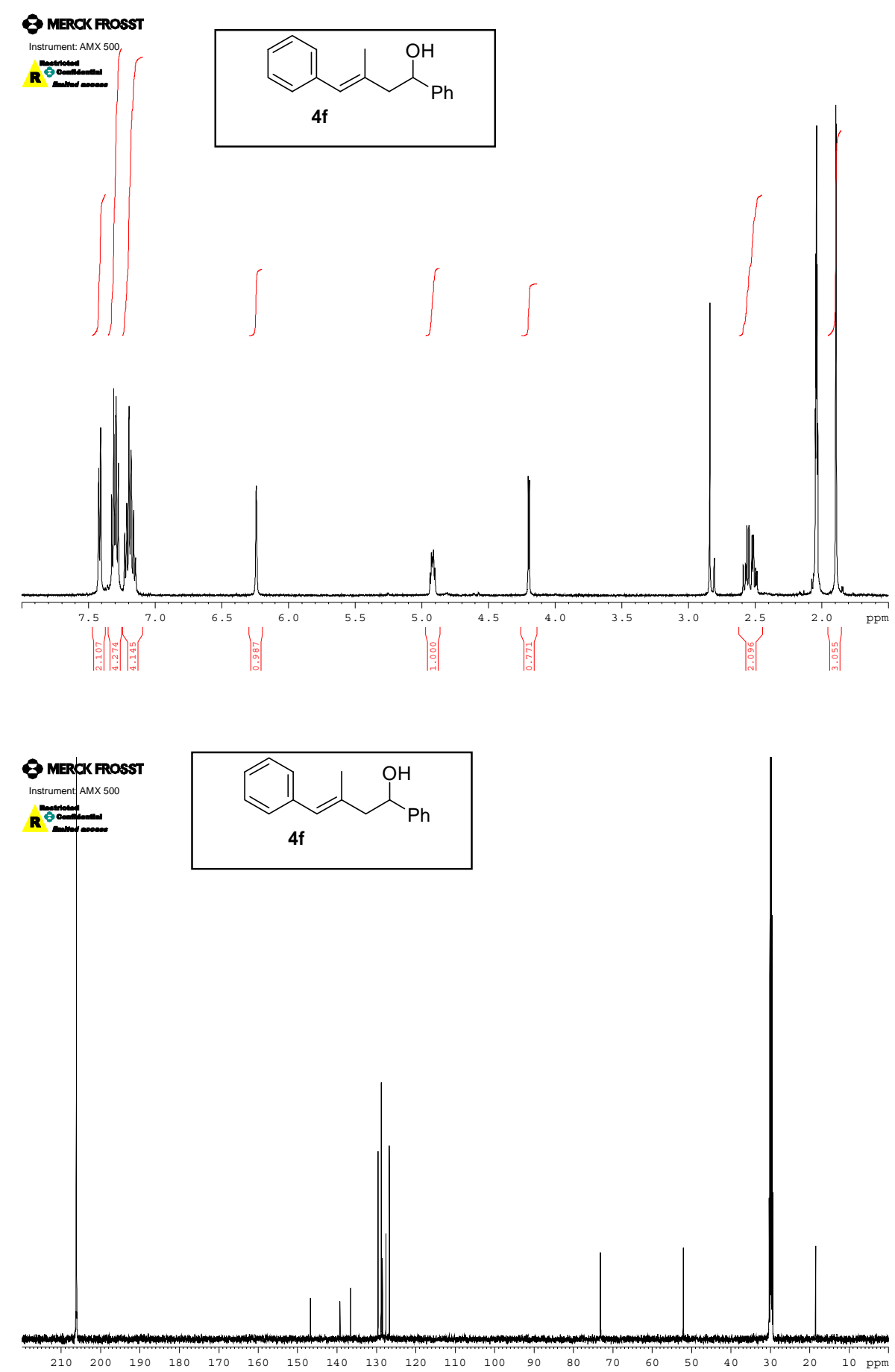

and Its Pd-Catalyzed Cross-Coupling under Mild Conditions

By Lianhai Li* and Neenah Navasero
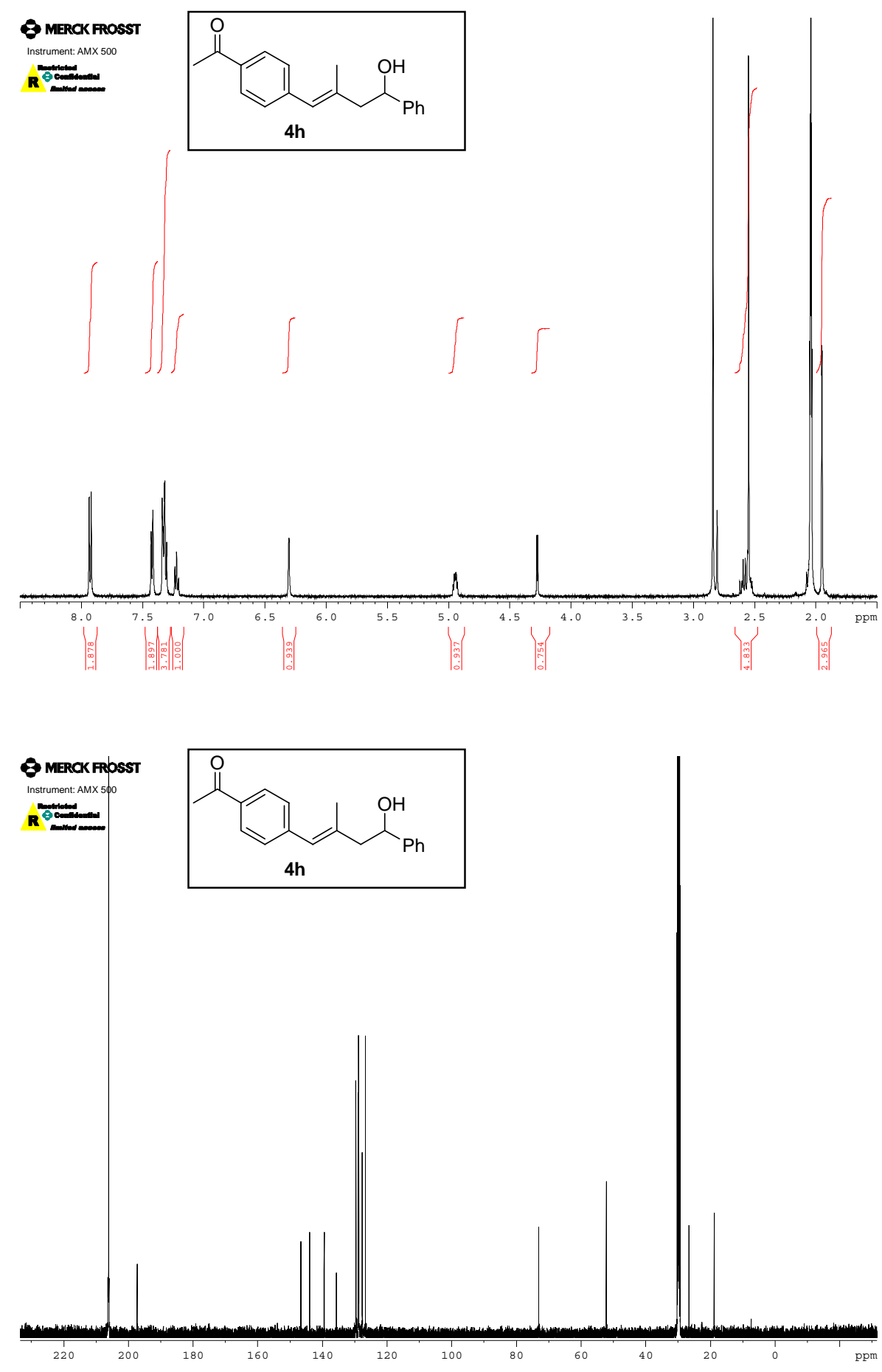

and Its Pd-Catalyzed Cross-Coupling under Mild Conditions

By Lianhai Li* and Neenah Navasero
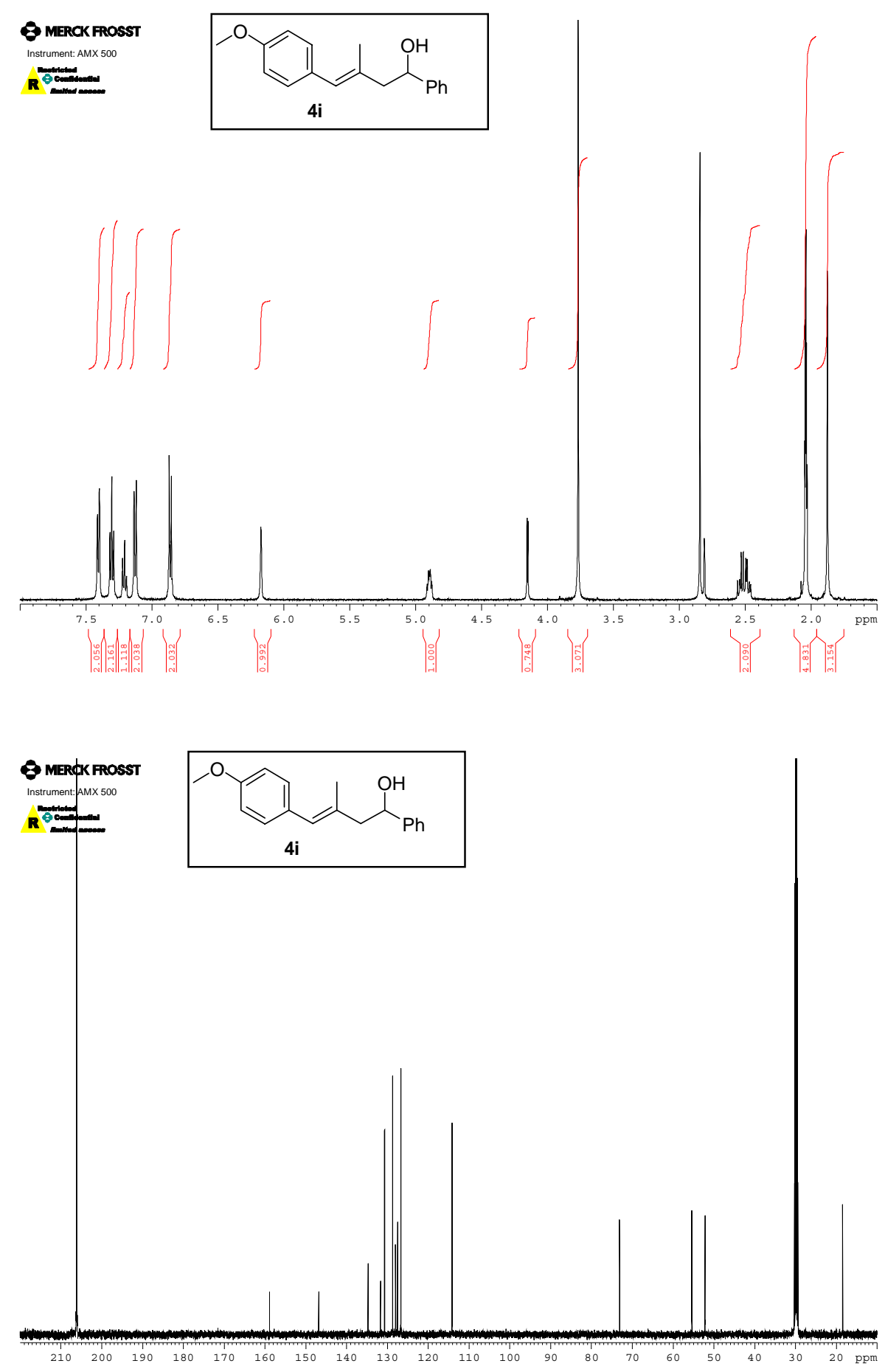

and Its Pd-Catalyzed Cross-Coupling under Mild Conditions

By Lianhai Li* and Neenah Navasero
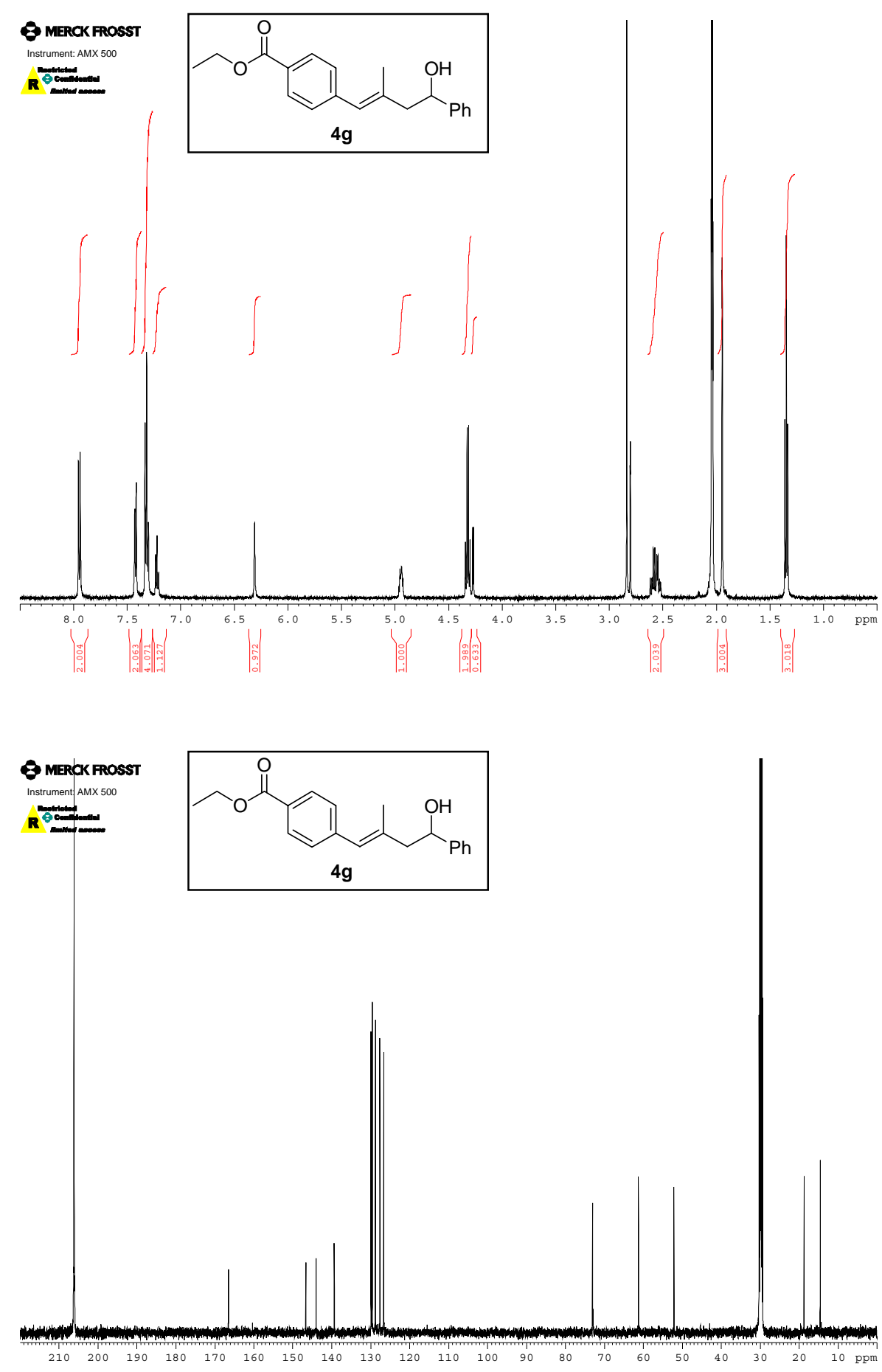

and Its Pd-Catalyzed Cross-Coupling under Mild Conditions

By Lianhai Li* and Neenah Navasero
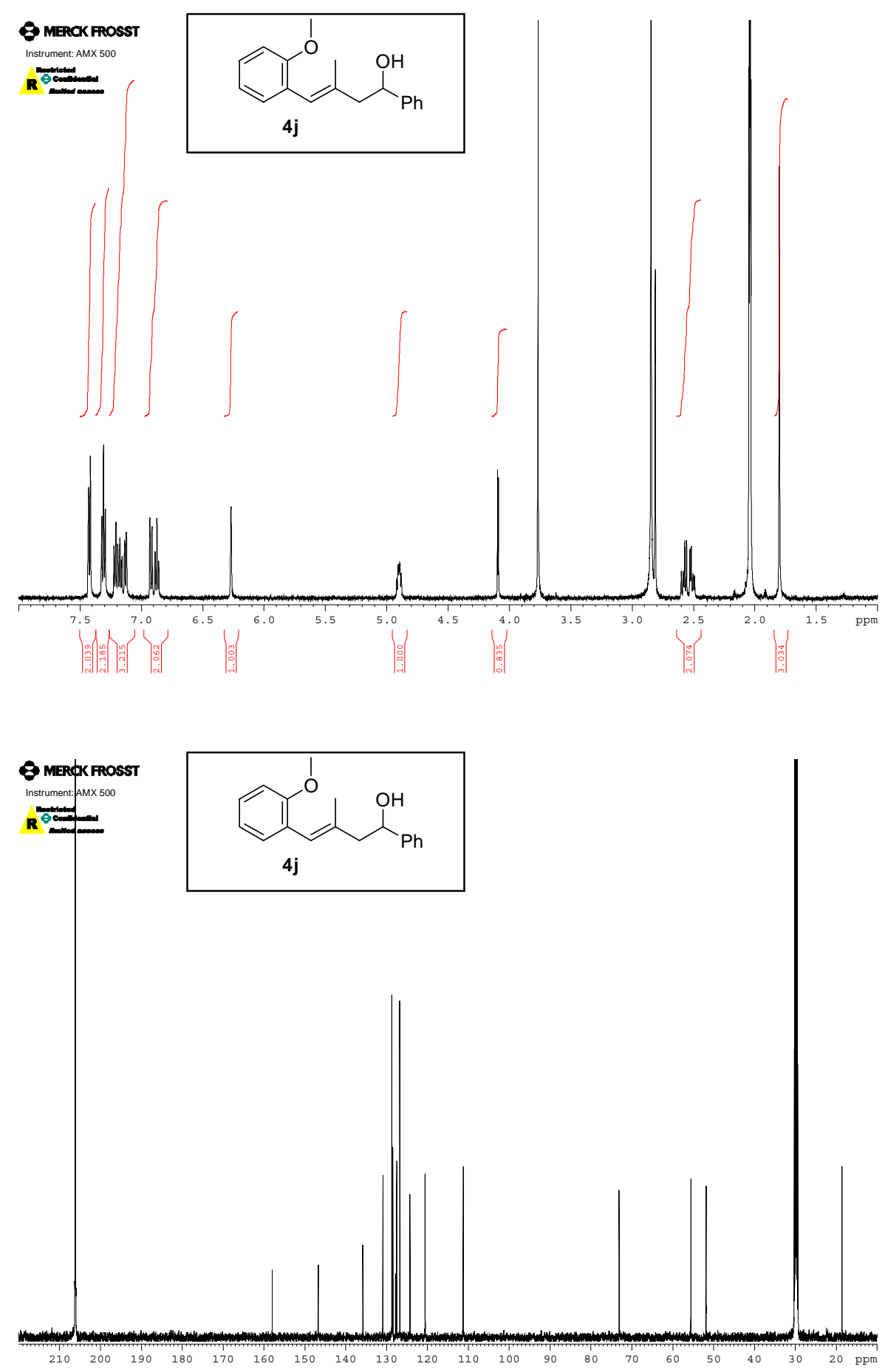